\title{
Time-dependent rate of multicomponent dark matter: Reproducing the DAMA/LIBRA phase- 2 results
}

\author{
Juan Herrero-Garcia, ${ }^{*}$ Andre Scaffidi, ${ }^{\dagger}$ Martin White, ${ }^{\ddagger}$ and Anthony G. Williams ${ }^{\S}$ \\ ARC Centre of Excellence for Particle Physics at the Terascale, Department of Physics, \\ University of Adelaide, Adelaide, South Australia 5005, Australia
}

(Received 25 September 2018; published 11 December 2018)

\begin{abstract}
The current paradigm for dark matter direct detection is to assume that the dark sector is solely composed of a single particle species. In this short paper, we make the observation that dark matter comprising both a light and a heavy component that modulate out of phase leads to interesting phenomenology in annual modulation experiments. For an illustrative example, we use the recently released DAMA/LIBRA phase-2 results with a lower energy threshold. Immediately after, it was argued that a one-component spinindependent dark matter explanation of the observed annual modulation is strongly disfavored or excluded unless isospin-violating couplings are invoked. We show that a simple two-component extension can reproduce the observed spectrum without the need to invoke fine-tuned couplings. Using the publicly available DAMA/LIBRA data, we perform a fit of the DAMA/LIBRA energy spectrum of the annual modulation amplitude to a scenario with two dark matter components. We also take into account how gravitational focusing affects the phases of the light and a heavy components differently, which leads to nontrivial effects in the total time-dependent rate. Our results show that there exists a unique solution in agreement with the data in the simplest case of isospin-conserving couplings with equal cross sections. The distinctive features found in this work are crucial for a dark matter interpretation of any observed annual modulation.
\end{abstract}

DOI: 10.1103/PhysRevD.98.123007

\section{INTRODUCTION}

Dark matter (DM) is one of nature's greatest enigmas. Until now, evidence for its existence has stemmed from its gravitational interactions. However, it is by no means guaranteed that just a single state or particle (1DM) constitutes the whole dark sector, which may have a multicomponent nature similarly to the visible sector. Multicomponent DM in direct detection has only been studied in a few works, which have then only focused on time-averaged (not modulated) event rates [1-8]. A generic prediction of two-component dark matter (2DM) is the presence of kinks in the differential event rates in monotarget experiments due to the different DM components [8]. In the following study, we observe another interesting prediction of $2 \mathrm{DM}$ in annual modulation

\footnotetext{
*juan.herrero-garcia@ coepp.org.au

andre.scaffidi@adelaide.edu.au

¥martin.white@adelaide.edu.au

§anthony.williams@adelaide.edu.au
}

Published by the American Physical Society under the terms of the Creative Commons Attribution 4.0 International license. Further distribution of this work must maintain attribution to the author(s) and the published article's title, journal citation, and DOI. Funded by SCOAP ${ }^{3}$. experiments, namely, that a light and heavy component will modulate out of phase, producing nontrivial modulation amplitudes that significantly affect the interpretation of the results. To study this effect, we adopt a purely phenomenological approach to try to reproduce the modulated signal observed by the DAMA/LIBRA Collaboration $[9,10]$ (denoted by DAMA in the following). We do so without going into further detail regarding the model building, which would affect the interactions and the abundances of the DM components. This is, in fact, studied for averaged rates in Ref. [11]. In this work, we also include gravitational focusing (GF) [12-14], which has a nontrivial effect on the 2DM time dependence of the direct detection rates and therefore on the modulation amplitude and phase. Although in our work we use the DAMA data, the physics discussed and the results obtained in the following apply to generic time-dependent signals of multicomponent scenarios.

Very recently, the DAMA Collaboration released the long-awaited phase- 2 results with a lower energy threshold, with two new energy bins below 2 electron equivalent energy (keVee) $[15,16]$. As first pointed out in version 3 of Ref. [17] and studied in Ref. [18] (see also Ref. [19]), the consistency of the DM interpretation of DAMA's signal is now under question, both for the light and the 
heavy DM mass solutions mentioned above for vanilla isospin-conserving spin-independent (SI) interactions, even before considering its compatibility with other null-result experiments. This is because below 2 keVee the direct detection rates for the two standard DM solutions behave very differently with decreasing recoil energy: the light DM gives rise to scatterings off iodine, increasing its rate significantly, while for the heavy DM (that scatters predominately off iodine), the modulation amplitude decreases, eventually giving rise to a phase flip. This was already pointed out in Ref. [20]. We confirm in this work that for SI interactions the energy spectrum for the $1 \mathrm{DM}$ is disfavored. Indeed, we find that the heavy solution is excluded at an even higher significance level when GF effects are accounted for.

The current significance for the modulation in the complete DAMA data set is $12.9 \sigma$. The DAMA Collaboration as well as several independent studies have not found that modulated backgrounds like those of neutrons, muons, solar neutrinos, or radon can be responsible for the signal [21-26]. Until the very latest phase-2 results, under reasonable particle physics and astrophysical assumptions, the signal was consistent in both amplitude and phase with that expected from weakly interacting massive particles (WIMPs). Assuming the standard halo model (SHM) and elastic scattering, the best-fit masses (and cross sections) are well known: a light DM with mass $\sim 10 \mathrm{GeV}$ scattering mainly on sodium (light mass solution) or a heavy DM with mass $\sim 70 \mathrm{GeV}$ scattering mainly on iodine (heavy mass solution) [27-34]. We show that the modulation observed by DAMA at low energies can be reproduced in a natural way by a combination of two DM particles, without the need to invoke fine-tuned isospinviolating couplings. ${ }^{1}$

The main issue with a DM interpretation of the DAMA modulation is that there is currently no accepted explanation that reconciles DAMA's signal with the absence of a positive signal in all other experiments [32,36-39], even independently of the DM velocity distribution [40-46]. This has motivated a large experimental effort to try to reproduce the DAMA experiment with $\mathrm{NaI}$ crystals in order to independently either confirm or reject its results [47-51]. The SABRE experiment [49] plans to have a northern and southern hemisphere pair of NaI detectors to search for a seasonal correlation or anticorrelation of any DAMA-like modulation signal [52]. Interestingly, the COSINUS experiment [50] aims to also measure the constant rate by developing a cryogenic detector. A null result in the

\footnotetext{
${ }^{1}$ We note that there have been studies that show that other effective operators can relieve the DAMA self-tension [19]. Also, the same authors claim that compatibility with other null results can be achieved in a protonphilic spin-dependent inelastic scenario when the DM velocity distribution departs from Maxwellian [35].
}

latter experiment may rule out a DM explanation of DAMA model independently [17].

This paper is structured as follows. In Sec. II, we introduce the relevant notation to describe the DM time-dependent signal in direct detection experiments. In Sec. III, we fit the binned amplitude of the DAMA modulation. We do this by generating pseudomock data from a modulating 2DM signal, including GF, and extracting the modulation amplitude by fitting a sinusoid to the resulting time-dependent signal. This allows us to draw conclusions regarding the effect of GF and the nonsinusoidal component. In this section, we also conduct the analysis for a single light/heavy DM to compare with results from other studies. We give our conclusions and final remarks in Sec. IV.

\section{DARK MATTER DIRECT DETECTION SIGNAL}

In this section, we present the relevant expressions for the direct detection of 2DM, with individual DM masses $m_{1,2}$ (we take $m_{1}<m_{2}$ ), SI cross-sections with protons $\sigma_{1,2}^{p}$, and local energy densities $\rho_{1,2}$. We use $\rho_{1}+\rho_{2}=\rho_{\text {loc }}$, where $\rho_{\text {loc }}$ is the local DM mass density. We take the astrophysical values of Ref. [8] and use the notation $r_{\rho} \equiv$ $\rho_{2} / \rho_{1}$ and $r_{\sigma} \equiv \sigma_{2}^{p} / \sigma_{1}^{p}$.

For 2DM with SI interactions, we can parametrize the differential event rate produced on a target nucleus with mass number $\mathrm{A}$ as [8]

$$
\begin{aligned}
\mathcal{R}_{A}\left(E_{R}, t\right)= & \frac{x_{A} \rho_{\mathrm{loc}} \sigma_{1}^{p}}{2\left(1+r_{\rho}\right) \mu_{p 1}^{2}} A^{2} F_{A}^{2}\left(E_{R}\right) \\
& \times\left[\frac{\eta\left(v_{m, A}^{(1)}, t\right)}{m_{1}}+\frac{r_{\rho} r_{\sigma} \mu_{p 1}^{2}}{\mu_{p 2}^{2}} \frac{\eta\left(v_{m, A}^{(2)}, t\right)}{m_{2}}\right],
\end{aligned}
$$

where here we define the halo integral as

$$
\eta\left(v_{m, A}^{(\alpha)}, t\right)=\int_{v>v_{m, A}^{(\alpha)}} d^{3} v \frac{f_{\mathrm{det}}^{(\alpha)}(\vec{v}, t)}{v} .
$$

From kinematics, the DM particle $\alpha(\alpha=1,2)$ is required to have a velocity larger than $v_{m, A}^{(\alpha)}=\sqrt{m_{A} E_{R} /\left(2 \mu_{\alpha A}^{2}\right)}$ to produce a recoil of energy $E_{R} \cdot m_{A}$ is the mass of the nucleus, $x_{A}$ is its mass faction in the detector, $\mu_{\alpha A}$ is the reduced DM-nucleus mass, and $F_{A}\left(E_{R}\right)$ is its nuclear form factor, for which we use the Helm parametrization [53,54]. In Eq. (2), $f_{\text {det }}^{(\alpha)}(\vec{v}, t)$ describes the distribution of DM particle velocities in the detector rest frame. In the following, we use the SHM, i.e., a Maxwellian velocity distribution, with equal velocity dispersions for both DM components (see Ref. [11] for a study regarding this assumption). As before, the total rate is given by the sum of the contributions on each target nucleus, i.e., $\mathcal{R}\left(E_{R}, t\right)=\sum_{A} \mathcal{R}_{A}\left(E_{R}, t\right)$. The rate for $1 \mathrm{DM}$ is obtained from Eq. (1) in the limit $r_{\rho}=0$. 
In this work, we deal with the time-dependent signal caused by the motion of the Earth around the Sun $[55,56]$. The total differential event rate can be decomposed as (see, for instance, Refs. [34,43,44,57])

$$
\mathcal{R}_{A}^{(\alpha)}\left(E_{R}, t\right) \equiv \bar{R}_{A}^{(\alpha)}\left(E_{R}\right)+\mathcal{M}_{A}^{(\alpha)}\left(E_{R}, t\right),
$$

where $\bar{R}_{A}^{(\alpha)}$ is the time-averaged rate and $\mathcal{M}_{A}^{(\alpha)}\left(E_{R}, t\right)$ is the time-dependent signal. In the following, we assume that all the time dependence stems from the velocity of the Earth $v_{e}(t)$, which is a reasonable assumption for the time scales of direct detection experiments. For isotropic and sufficiently smooth DM halos, to leading order on $v_{e}(t),{ }^{2}$ the time-dependent signal consists of the annual modulation,

$$
\mathcal{M}_{A}^{(\alpha)}\left(E_{R}, t\right)=\mathcal{M}_{A}^{(\alpha)}\left(E_{R}\right) \cos \left[2 \pi\left(t-t_{0}\left(v_{m, A}^{(\alpha)}\right)\right],\right.
$$

where $t_{0}\left(v_{m, A}^{(\alpha)}\right)$ is the phase of the modulation and

$\mathcal{M}_{A}^{(\alpha)}\left(E_{R}\right)=\frac{1}{2}\left[\mathcal{M}_{A}^{(\alpha)}\left(E_{R}, t_{\mathrm{J}}\right)-\mathcal{M}_{A}^{(\alpha)}\left(E_{R}, t_{\mathrm{J}}+0.5\right)\right]$

is the amplitude. $t_{\mathrm{J}}\left(t_{\mathrm{J}}+0.5\right)$ measured in years corresponds to June (December) 1, which for minimum velocities $v_{m, A}^{(\alpha)}$ above $\sim 200 \mathrm{~km} \mathrm{~s}^{-1}$ is the time of the year when the velocity of the WIMP flow in the Earth's frame is maximum (minimum). For $v_{m, A}^{(\alpha)}<200 \mathrm{~km} \mathrm{~s}^{-1}$, the situation is the opposite: $t_{\mathrm{J}}\left(t_{\mathrm{J}}+0.5\right) t_{\mathrm{J}}\left(t_{\mathrm{J}}+0.5\right)$ corresponds to minimum (maximum) WIMP flow, such that $\mathcal{M}_{A}^{(\alpha)}\left(E_{R}\right)$ becomes negative. In other words, for small enough $v_{m, A}^{(\alpha)}$, the phase of the modulation flips by 6 months. As we show below, this is precisely what happens for heavy DM components scattering off iodine in DAMA at the lowest energies. The total rates are given by the sum over nuclei and DM components, $\bar{R}\left(E_{R}\right)=\sum_{\alpha, A} \bar{R}_{A}^{(\alpha)}\left(E_{R}\right)$ and $\mathcal{M}\left(E_{R}, t\right)=$ $\sum_{\alpha, A} \mathcal{M}_{A}^{(\alpha)}\left(E_{R}, t\right)$.

\section{A. Gravitational focusing and the nonsinusoidal signal}

GF of DM particles by the Sun affects the phase of the modulation $t_{0}$, which, for an observed $E_{R}$, is sensitive to the DM mass via the dependence on $v_{m, A}^{(\alpha)}$ [12-14]. The effect is significant for $v_{m, A}^{(\alpha)} \lesssim 200 \mathrm{~km} \mathrm{~s}^{-1}$, in which case the phase is changed from the vanilla case of December 1 toward a later value. In the case of DAMA, the effect on the phase is important for heavy DM masses, for which it can change by tens of days. For light DM particles, the effect is negligible, and the phase remains at June 1. In 2DM, the most interesting feature is that the sum of the time-dependent

\footnotetext{
${ }^{2} \mathrm{~A}$ nonsinusoidal modulation is expected when higher-order harmonics, which are, however, suppressed by extra powers of $v_{e}$, are considered $[13,14,21]$.
}

signals of the individual components, with their phases $t_{0}^{(1,2)}$ being different due to GF $\left(t_{0}^{(2)}>t_{0}^{(1)}\right.$ as $\left.m_{2}>m_{1}\right)$, leads to a nonsinusoidal time-dependent signal only suppressed by the (small) phase difference of the 2DM components $\Delta t_{0} \equiv t_{0}^{(2)}-t_{0}^{(1)}>0$.

We can see this by expanding the combined signal in $\Delta t_{0}$ (see also Ref. [57]). For a given nuclei and recoil energy, $\Delta t_{0}$ depends on the mass splitting of both DM components. We find that the combined $\mathcal{M}\left(E_{R}, t\right)$ is given to leading order in $\Delta t_{0}$ by

$$
\begin{aligned}
\mathcal{M}(t)= & \mathcal{M}^{(1)} \cos \left[2 \pi\left(t-t_{0}^{(1)}\right)\right]+\mathcal{M}^{(2)} \cos \left[2 \pi\left(t-t_{0}^{(1)}-\Delta t_{0}\right)\right] \\
= & \left(\mathcal{M}^{(1)}+\mathcal{M}^{(2)}\right) \cos \left[2 \pi\left(t-t_{0}^{(1)}\right)\right] \\
& +\mathcal{M}^{(2)}\left(\frac{\Delta t_{0}}{t-t_{0}^{(1)}}\right) \sin \left[2 \pi\left(t-t_{0}^{(1)}\right)\right]
\end{aligned}
$$

where the expansion is valid for $t \gg t_{0}^{(2)}>t_{0}^{(1)}$. For $t \sim t_{0}^{(1,2)}$, higher-order terms become important. As can be observed, the sine term is proportional to $\Delta t_{0}$. Therefore, for non-negligible $\Delta t_{0}$, the total time-dependent signal is nonsinusoidal. For the DAMA observation, how good this approximation is depends on the DM masses, which we study in the following by fitting the DAMA phase-2 timedependent signal to the numerically computed one using Eq. (1) with GF effects implemented.

\section{DAMA ENERGY SPECTRUM OF THE MODULATION AMPLITUDE}

We perform our analysis on the whole energy spectrum of the DAMA annual modulation amplitude, which combines results from DAMA/NaI and DAMA/LIBRA phases 1 and 2 . The total exposure is $2.46 \mathrm{~kg} \cdot \mathrm{y}$. The events are measured in electron equivalent energy, which is related to the true recoil energy $E_{R}$ through the target-dependent quenching factors $E_{\mathrm{ee}}=Q_{A} E_{R}$ (we use $Q_{\mathrm{Na}}=0.3$ and $\left.Q_{\mathrm{I}}=0.09\right)$. We employ the differential response function of Ref. [58], treating the parameters $\alpha_{\mathrm{LE}}=(0.448 \pm$ $0.035) \sqrt{\mathrm{keVee}}$ and $\beta_{\mathrm{LE}}=(9.1 \pm 5.1) \times 10^{-3}$ as nuisance parameters. The results made public by the DAMA Collaboration are presented in slide 22 of Ref. [15]. We use the data of Table I of Ref. [18], which gives the observed binned annual modulation amplitude $M_{i}$ in $N=$ 10 bins in the energy range $[1,20]$ keVee in order to increase the signal-to-noise ratio in our fit.

We undertake our analysis as follows:

(1) We first generate pseudomock data from a modulating DM signal with gravitational focusing corrections implemented and 2.46 ton y of exposure in the time intervals used by DAMA. The term "pseudomock" here refers to the fact that we use the "Asimov data," which in the large statistics limit correspond to the expected Poisson mean in each time bin. This is done 
TABLE I. Results of 1DM fit to the DAMA energy spectrum after accounting for GF corrections. The different fits are for both the light and the heavy DM mass solutions. The dashes refer to the parameters that are fixed to 1 .

\begin{tabular}{lccccc}
\hline \hline & \multicolumn{5}{c}{$\sigma_{1}^{p}$} \\
1DM & $m(\mathrm{GeV})$ & $\left(\times 10^{-40} \mathrm{~cm}^{2}\right)$ & $\chi_{\min }^{2} / \mathrm{dof}$ & $\mathrm{p}$ value & $\mathcal{Z}$ \\
\hline Light & 8.42 & 1.25 & $48.4 / 8$ & $8.15 \times 10^{-8}$ & 5.40 \\
Heavy & 70.1 & 0.10 & $56.8 / 8$ & $1.94 \times 10^{-9}$ & 6.00 \\
\hline \hline
\end{tabular}

TABLE II. Same as Table I for 2DM fit to the DAMA energy spectrum.

\begin{tabular}{lccccccc}
\hline \hline 2DM & $m_{1}$ & $m_{2}$ & $\sigma_{1}^{p}$ & $r_{\rho}$ & $\chi_{\min }^{2} / \mathrm{dof}$ & $\mathrm{p}$ value & $\mathcal{Z}$ \\
\hline$r_{\sigma}=1$ & 22.0 & 80.3 & 0.14 & 3.35 & $11.8 / 6$ & 0.07 & 1.84 \\
\hline
\end{tabular}

to ensure smooth likelihood functions in our statistical analyses. We only generate signal events, since the DAMA Collaboration releases no information about the observed constant backgrounds.

(2) As DAMA does, we then fit the function $R_{A}(t)=$ $S_{0}+S_{m} \cos \left[2 \pi\left(t-t_{0}\right) / T\right]$ to the pseudomock data set in each energy bin. Since the DAMA experiment does not veto backgrounds, we fit a sinusoidal component plus a constant offset to extract the bestfit modulation amplitude $S_{m}$. We take a constant phase of $t_{0}=152.5$ days and a period $T=1 \mathrm{yr}^{3}$

(3) We take the estimated amplitude for each energy bin and compare these to the DAMA data using a maximum likelihood analysis in order to extract the goodness of fit and best-fit values of the 1DM and 2DM model parameters.

The reason for this roundabout approach is that the DAMA Collaboration does not release time-dependent data in $0.5 \mathrm{keVee}$ bins, ${ }^{4}$ and so we proceed as closely as possible to the method used by the collaboration to extract the modulation amplitude. This would not be necessary if we did not consider GF, but GF nontrivially changes the phase of the modulation away from June 1 as well as the shape of the time-dependent signal.

We use the open source software Minuit [59] to compute the best-fit points, which we give in Tables I

\footnotetext{
${ }^{3}$ The DAMA Collaboration also presents results assuming variable phases and additional nonsinusoidal components. Energy spectra for these fits, however, are presented in larger integrated energy bins of 1 keVee width. Since any interesting behavior in the spectrum occurs in the lowest [1-1.5] keVee energy bin, we do not use these other data sets.

${ }^{4}$ The only time-dependent information released by the DAMA Collaboration is residuals in large integrated energy bins [1-3], [2-6], and [1-6] keVee. However, in the latter, the energy information of the (most interesting) lowest energy bins is washed out.
}
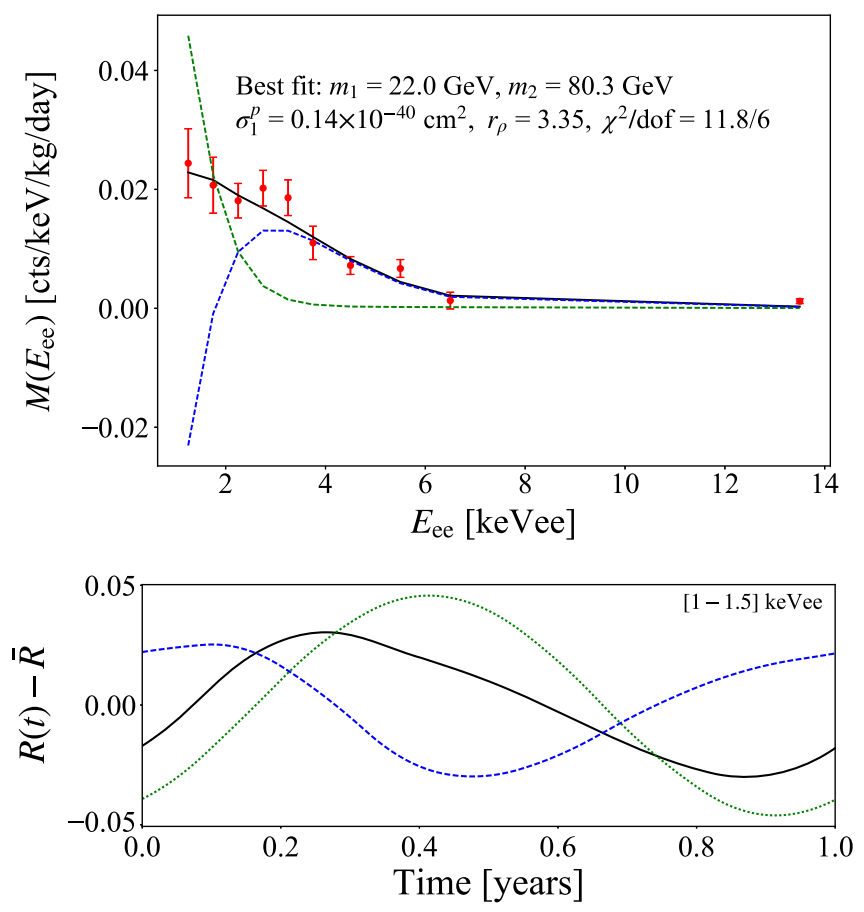

FIG. 1. Two-component fit to the DAMA/LIBRA phase-2 data. We also show the contribution from DM1 (DM2) in dotted green (dashed blue) and the combined (solid black). Upper panel: Bestfit spectra of the DAMA energy spectrum for the 2DM model. The experimental best-fit modulation amplitudes to the combined DAMA phases 1 and 2 rebinned [18] are shown with red points. Below the phase flip, which occurs at $\sim 2 \mathrm{keVee}$, the contributions of the two components partially neutralize each other in the combined modulation. Lower panel: Time-dependent residuals in the lowest energy bin, [1-1.5] keVee. Notice the antimodulation of DM2 and the nonsinusoidality of DM2 and the combined signal.

and II. For completeness, we also compute the corresponding $\mathrm{p}$ values for the fits, as well as the corresponding number of equivalent two-sided Gaussian standard deviations $\mathcal{Z}$. We deem a "good fit" to be one that gives a $\mathrm{p}$ value $p>0.05$ that corresponds to $\mathcal{Z}<1.96$. For $2 \mathrm{DM}$, we also employ the MultiNest implementation of the nested sampling algorithm [60-62], with 5000 live points and a tolerance of 0.5 . We then determine the distribution of the profile likelihood ratio (PLR) $\mathcal{L} / \mathcal{L}_{\max }$ throughout the parameter space from the obtained samples.

\section{A. One-component dark matter fit}

In Table I, we show results of 1DM fits for the light and the heavy DM mass solutions, which correspond to scatterings mainly in $\mathrm{Na}$ and I, respectively. We also check the exclusion significances for the 1DM case neglecting gravitational focusing effects to check consistency with previous studies. Since GF does not affect the sinusoidality of the DAMA signal for DM masses below $\sim 30 \mathrm{GeV}$, we find that the light solution is excluded at $5.4 \sigma$, which is 

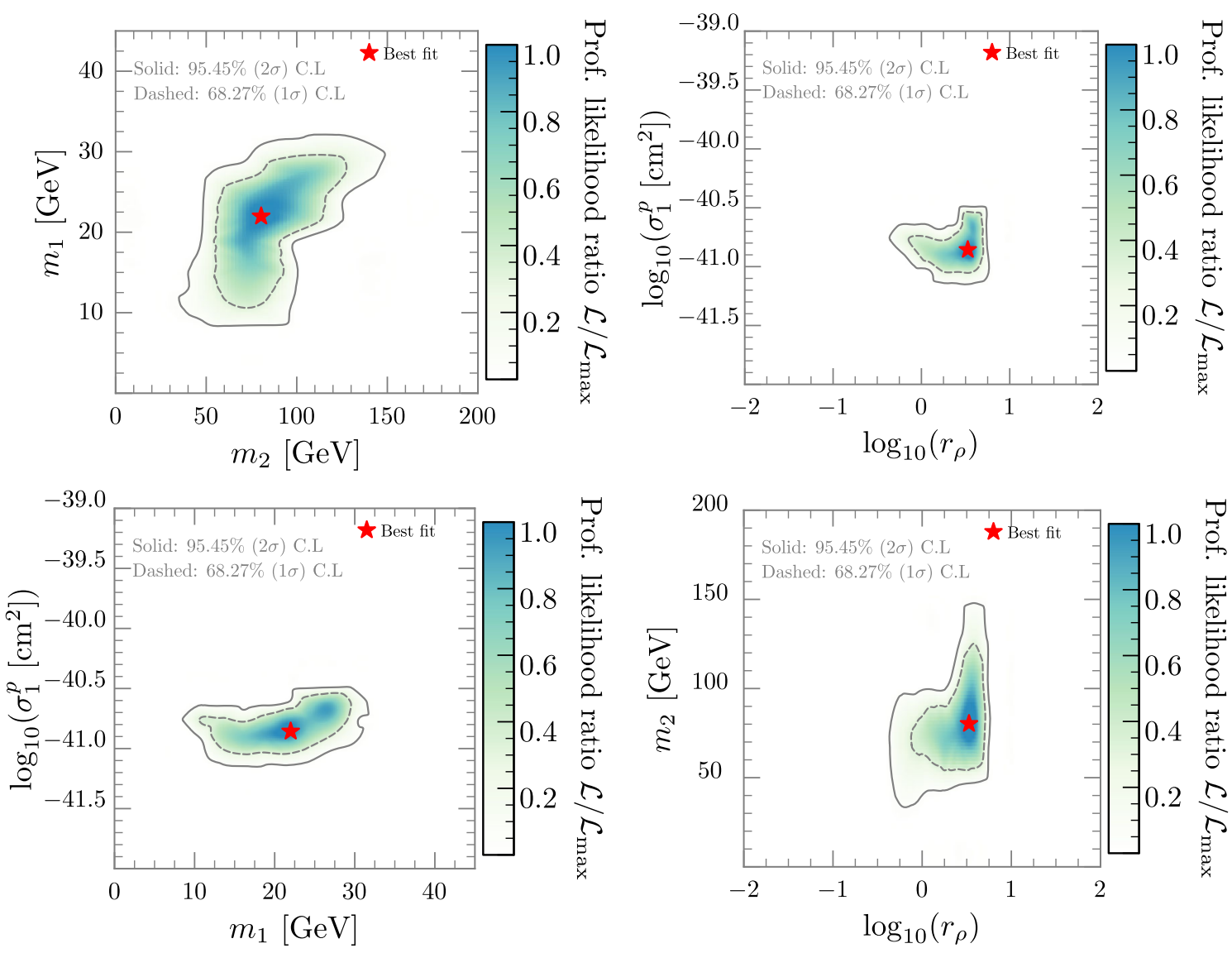

FIG. 2. PLR density overlaid with 1 and $2 \sigma$ C.L. contours for the 2DM fit to the DAMA energy spectrum of the modulation amplitude as calculated in Sec. III B. We indicate the best-fit points with a red star (see Table II.)

roughly the same significance as observed in previous studies. Interestingly, however, we see that including GF corrections nontrivially increases the exclusion significance of the heavy solution from $\sim 3 \sigma$ to $6 \sigma$.

\section{B. Two-component dark matter fit}

We show in Table II the result of the fit for 2DM. We fix $r_{\sigma}=1$, leaving $r_{\rho}$ free. ${ }^{5}$ One can observe that the $2 \mathrm{DM}$ model provides a good fit with an exclusion significance of only $1.84 \sigma$. In Fig. 1 (upper panel), we show the binned modulation amplitude (solid black) for the best-fit points of the 2DM solution. This corresponds to scattering of both DM components dominantly off iodine. The fact that the lighter component scatters dominantly off iodine with a negligible contribution from sodium is due to two factors: first, the smaller quenching factor in iodine compensates its larger mass, translating into smaller $v_{\mathrm{m}, \mathrm{I}}^{(1)}\left(E_{R}\right)$ and thus into larger $\eta\left(v_{\mathrm{m}, \mathrm{I}}^{(1)}\right)$; second is the $A^{2}$ enhancement factor for

\footnotetext{
${ }^{5}$ The energy density and the cross section enter identically in the rate. Therefore, apart from the overall normalization, one can fix $r_{\sigma}$ and consider $r_{\rho}$ as a free parameter.
}

iodine due to coherent SI scatterings. Below 2 keVee, 2DM becomes negative (i.e., a phase flip), and 1DM dominates the total rate. Therefore, there is a partial cancellation in the combined modulation between the individual DM contributions. This is illustrated in Fig. 1 (lower panel), in which we plot the time-dependent residual for 2DM in the lowest recoil energy bin $[1-1.5]$ keVee. $^{6}$

In Fig. 2, we present the frequentist 1 and $2 \sigma$ C.L. contours using PIPPI [63]. We show the planes $m_{1}-m_{2}$ (top left), $\log _{10}\left(r_{\rho}\right)-\log _{10}\left(\sigma_{1}^{p}\right)$ (top right), $\log _{10}\left(r_{\rho}\right)-m_{2}$ (bottom left), and $\log _{10}\left(r_{\rho}\right)-m_{2}$ (bottom right). The regions are very well defined around the best-fit values. The $2 \sigma$ range of $m_{1}$ is quite narrow, [8,30] GeV, while that of $m_{2}$ is larger, $[30,150] \mathrm{GeV}$; i.e., there is more freedom in the heavy component since for heavier DM the dependence on the mass via $\eta\left(v_{m, A}^{(\alpha)}\right)$ is milder. For large mass values, $m_{1}$ and

\footnotetext{
${ }^{6}$ For completeness, we have also conducted a similar analysis without including GF. In this case, we obtain two solutions: one that corresponds to a similar one as with GF and another one at $m_{1} \sim 8 \mathrm{GeV}$ and $m_{2} \sim 170$ with $r_{\rho} \sim 0.07$. That is, the second solution prefers a suppressed heavy component, i.e., a 1DM scenario.
} 
$m_{2}$ show a mild positive correlation, such that both individual terms in the square bracket of Eq. (1) become similar in size. Moreover, one can observe how the largest $m_{2}$ region corresponds to large $r_{\rho}$ (bottom right panel), which is easily understood looking at the second term in the square bracket of Eq. (1). Also, the region of large $\sigma_{1}^{p}$ corresponds to large $r_{\rho}$, see the top left panel, as expected from the overall normalization of the rate.

\section{CONCLUSIONS}

In this paper, we have studied how a simple 2DM can lead to interesting signals in annual modulation experiments. Furthermore, we showed that implementing corrections due to GF leads to nonsinusoidal and nontrivial phase effects in the evaluation of the 2DM time-dependent rate. For an illustrative example, we studied whether a 2DM model comprising both a light and heavy component can revive the vanilla DM interpretation of the low-threshold DAMA data. We performed a fit of 2DM to the publicly available DAMA data using the energy spectrum of the amplitude of the modulation. For the first time, we also fully incorporate gravitational focusing effects into such an analysis. First, we fit 1DM to the energy spectrum data and find that the heavy solution is even more excluded than shown in previous studies after GF effects are considered. On the contrary, we find that $2 \mathrm{DM}$ provides very good agreement to the energy spectrum data.

We show our solution in Fig. 1, in which scatterings are predominantly off iodine, with a crossing between the spectrum of the two individual DM components at roughly 2 keVee. The crossing occurs due to a phase flip in the heavier component at low recoil energy. The results of the 2DM fits are summarized in Table II and Figs. 1 and 2, which involve reasonable values of the relative energy densities and the cross section. The key feature found in the analysis is that, at the lowest energies, the modulation of the heavy DM particle becomes negative, so in the combined modulation, the individual DM contributions partially cancel each other.

Therefore, two-component DM looks like a natural solution to the first part of the DAMA puzzle: the compatibility of the spectrum with that expected from DM under standard astrophysical and particle physics assumptions. The second part of the DAMA puzzle, that is, the compatibility of DAMA data with other null results, is not solved.

We would like to conclude by saying that it would be very helpful if the DAMA Collaboration were to make public the temporal data in smaller energy bins, which would allow the use of all information. In particular, it would show whether or not there is a nonsinusoidal behavior in the current data in the $[1,1.5]$ keVee bin, as present in the case of 2DM (see the lower panel of Fig. 1).

In any case, even if the DAMA signal turns out not to be related to DM, the distinctive features of the timedependent signal of multicomponent DM found in this work, like the nonsinusoidal behavior and the possibility of having a partial cancellation at low energies, are generic predictions that should be searched for in case an annual modulation signal is observed in next-generation experiments.

\section{ACKNOWLEDGMENTS}

J. H. G. would like to thank Thomas Schwetz for useful discussions. This work is supported by the Australian Research Council through the Centre of Excellence for Particle Physics at the Terascale, Grant No. CE110001004. M. W. is supported by the Australian Research Council Future Fellowship, Grant No. FT140100244. M. W. wishes to thank Lucien Boland and Sean Crosby for their administration of, and ongoing assistance with, the MPI-enabled computing cluster on which this work was performed.
[1] S. Profumo, K. Sigurdson, and L. Ubaldi, Can we discover multi-component WIMP dark matter?, J. Cosmol. Astropart. Phys. 12 (2009) 016.

[2] B. Batell, M. Pospelov, and A. Ritz, Direct detection of multi-component secluded WIMPs, Phys. Rev. D 79, 115019 (2009).

[3] A. Adulpravitchai, B. Batell, and J. Pradler, Non-Abelian discrete dark matter, Phys. Lett. B 700, 207 (2011).

[4] K. R. Dienes, J. Kumar, and B. Thomas, Direct detection of dynamical dark matter, Phys. Rev. D 86, 055016 (2012).

[5] D. Chialva, P. S. B. Dev, and A. Mazumdar, Multiple dark matter scenarios from ubiquitous stringy throats, Phys. Rev. D 87, 063522 (2013).
[6] S. Bhattacharya, P. Poulose, and P. Ghosh, Multipartite interacting scalar dark matter in the light of updated LUX data, J. Cosmol. Astropart. Phys. 04 (2017) 043.

[7] S. Bhattacharya, P. Ghosh, T. N. Maity, and T. S. Ray, Mitigating direct detection bounds in non-minimal Higgs portal scalar dark matter models, J. High Energy Phys. 10 (2017) 088.

[8] J. Herrero-Garcia, A. Scaffidi, M. White, and A. G. Williams, On the direct detection of multi-component dark matter: Sensitivity studies and parameter estimation, J. Cosmol. Astropart. Phys. 11 (2017) 021.

[9] R. Bernabei et al. (DAMA and LIBRA Collaborations), New results from DAMA/LIBRA, Eur. Phys. J. C 67, 39 (2010). 
[10] R. Bernabei et al., Final model independent result of DAMA/LIBRA-phase1, Eur. Phys. J. C 73, 2648 (2013).

[11] J. Herrero-Garcia, A. Scaffidi, M. White, and A. G. Williams, On the direct detection of multi-component dark matter: Implications of the relic abundance, arXiv:1809 .06881 .

[12] M. S. Alenazi and P. Gondolo, Phase-space distribution of unbound dark matter near the Sun, Phys. Rev. D 74, 083518 (2006).

[13] S. K. Lee, M. Lisanti, A. H. G. Peter, and B. R. Safdi, Effect of Gravitational Focusing on Annual Modulation in DarkMatter Direct-Detection Experiments, Phys. Rev. Lett. 112, 011301 (2014).

[14] N. Bozorgnia and T. Schwetz, Is the effect of the Sun's gravitational potential on dark matter particles observable?, J. Cosmol. Astropart. Phys. 08 (2014) 013.

[15] D. C. R. Bernabei, LNGS Scientific Committee Meeting, https://agenda.infn.it/conferenceDisplay.py?confId=15474, 2018.

[16] R. Bernabei et al., First model independent results from DAMA/LIBRA-phase2, arXiv:1805.10486.

[17] F. Kahlhoefer, F. Reindl, K. Schäffner, K. Schmidt-Hoberg, and S. Wild, Model-independent comparison of annual modulation and total rate with direct detection experiments, J. Cosmol. Astropart. Phys. 05 (2018) 074.

[18] S. Baum, K. Freese, and C. Kelso, Dark matter implications of DAMA/LIBRA-phase2 results, arXiv:1804.01231.

[19] S. Kang, S. Scopel, G. Tomar, and J.-H. Yoon, DAMA/ LIBRA-phase2 in WIMP effective models, J. Cosmol. Astropart. Phys. 07 (2018) 016.

[20] C. Kelso, P. Sandick, and C. Savage, Lowering the threshold in the DAMA dark matter search, J. Cosmol. Astropart. Phys. 09 (2013) 022.

[21] S. Chang, J. Pradler, and I. Yavin, Statistical tests of noise and harmony in dark matter modulation signals, Phys. Rev. D 85, 063505 (2012).

[22] E. Fernandez-Martinez and R. Mahbubani, The Gran Sasso muon puzzle, J. Cosmol. Astropart. Phys. 07 (2012) 029.

[23] J. Klinger and V. A. Kudryavtsev, Can muon-induced backgrounds explain the DAMA data?, J. Phys. Conf. Ser. 718, 042033 (2016).

[24] P. Belli et al., Search for double beta decay in ${ }^{106} \mathrm{Cd}$ in the DAMA/CRYS setup, AIP Conf. Proc. 1894, 020005 (2017).

[25] R. Bernabei and F. Cappella, Investigation of rare nuclear decays with the DAMA set-ups, Int. J. Mod. Phys. A 33, 1843005 (2018).

[26] D. N. McKinsey, Is DAMA bathing in a sea of radioactive argon?, arXiv:1803.10110.

[27] A. Bottino, F. Donato, N. Fornengo, and S. Scopel, Light neutralinos and WIMP direct searches, Phys. Rev. D 69, 037302 (2004).

[28] A. Bottino, F. Donato, N. Fornengo, and S. Scopel, Lower bound on the neutralino mass from new data on CMB and implications for relic neutralinos, Phys. Rev. D 68, 043506 (2003).

[29] P. Gondolo and G. Gelmini, Compatibility of DAMA dark matter detection with other searches, Phys. Rev. D 71, 123520 (2005).
[30] M. Fairbairn and T. Schwetz, Spin-independent elastic WIMP scattering and the DAMA annual modulation signal, J. Cosmol. Astropart. Phys. 01 (2009) 037.

[31] J. Kopp, T. Schwetz, and J. Zupan, Global interpretation of direct dark matter searches after CDMS-II results, J. Cosmol. Astropart. Phys. 02 (2010) 014.

[32] T. Schwetz and J. Zupan, Dark matter attempts for CoGeNT and DAMA, J. Cosmol. Astropart. Phys. 08 (2011) 008.

[33] E. Del Nobile, G. B. Gelmini, A. Georgescu, and J.-H. Huh, Reevaluation of spin-dependent WIMP-proton interactions as an explanation of the DAMA data, J. Cosmol. Astropart. Phys. 08 (2015) 046.

[34] J. Herrero-Garcia, Halo-independent tests of dark matter annual modulation signals, J. Cosmol. Astropart. Phys. 09 (2015) 012.

[35] S. Kang, S. Scopel, G. Tomar, and J.-H. Yoon, Proton-philic spin-dependent inelastic dark matter (pSIDM) as a viable explanation of DAMA/LIBRA-phase2, arXiv:1810.09674.

[36] D. S. Akerib et al. (LUX Collaboration), Results from a Search for Dark Matter in the Complete LUX Exposure, Phys. Rev. Lett. 118, 021303 (2017).

[37] E. Aprile et al. (XENON Collaboration), First Dark Matter Search Results from the XENON1T Experiment, Phys. Rev. Lett. 119, 181301 (2017).

[38] X. Cui et al. (PandaX-II Collaboration), Dark Matter Results From 54-Ton-Day Exposure of PandaX-II Experiment, Phys. Rev. Lett. 119, 181302 (2017).

[39] C. Savage, G. Gelmini, P. Gondolo, and K. Freese, XENON10/100 dark matter constraints in comparison with CoGeNT and DAMA: Examining the Leff dependence, Phys. Rev. D 83, 055002 (2011).

[40] C. McCabe, DAMA and CoGeNT without astrophysical uncertainties, Phys. Rev. D 84, 043525 (2011).

[41] M. T. Frandsen, F. Kahlhoefer, J. March-Russell, C. McCabe, M. McCullough, and K. Schmidt-Hoberg, On the DAMA and CoGeNT modulations, Phys. Rev. D 84, 041301 (2011).

[42] M. T. Frandsen, F. Kahlhoefer, C. McCabe, S. Sarkar, and K. Schmidt-Hoberg, Resolving astrophysical uncertainties in dark matter direct detection, J. Cosmol. Astropart. Phys. 01 (2012) 024.

[43] J. Herrero-Garcia, T. Schwetz, and J. Zupan, On the annual modulation signal in dark matter direct detection, J. Cosmol. Astropart. Phys. 03 (2012) 005.

[44] J. Herrero-Garcia, T. Schwetz, and J. Zupan, Astrophysics Independent Bounds on the Annual Modulation of Dark Matter Signals, Phys. Rev. Lett. 109, 141301 (2012).

[45] N. Bozorgnia, J. Herrero-Garcia, T. Schwetz, and J. Zupan, Halo-independent methods for inelastic dark matter scattering, J. Cosmol. Astropart. Phys. 07 (2013) 049.

[46] G. B. Gelmini, J.-H. Huh, and S.J. Witte, Assessing compatibility of direct detection data: Halo-independent global likelihood analyses, J. Cosmol. Astropart. Phys. 10 (2016) 029.

[47] J. Amaré et al., From ANAIS-25 towards ANAIS-250, Phys. Procedia 61, 157 (2015).

[48] K. Fushimi et al. (PICO-LON Collaboration), Dark matter search project PICO-LON, J. Phys. Conf. Ser. 718, 042022 (2016). 
[49] E. Shields, J. Xu, and F. Calaprice, SABRE: A new NaI(T1) dark matter direct detection experiment, Phys. Procedia 61, 169 (2015).

[50] G. Angloher et al., The COSINUS project-Perspectives of a NaI scintillating calorimeter for dark matter search, Eur. Phys. J. C 76, 441 (2016).

[51] W. G. Thompson et al. (COSINE-100 Collaboration), Current status and projected sensitivity of COSINE-100, in Proceedings of 15th International Conference on Topics in Astroparticle and Underground Physics (TAUP 2017), Sudbury, Ontario, Canada (2017) [arXiv:1711.01488].

[52] F. Froborg et al. (SABRE Collaboration), SABRE: WIMP modulation detection in the northern and southern hemisphere, J. Phys. Conf. Ser. 718, 042021 (2016).

[53] J. Lewin and P. Smith, Review of mathematics, numerical factors, and corrections for dark matter experiments based on elastic nuclear recoil, Astropart. Phys. 6, 87 (1996).

[54] R. H. Helm, Inelastic and elastic scattering of $187-\mathrm{Mev}$ electrons from selected even-even nuclei, Phys. Rev. 104, 1466 (1956).

[55] A. K. Drukier, K. Freese, and D. N. Spergel, Detecting cold dark matter candidates, Phys. Rev. D 33, 3495 (1986).
[56] K. Freese, J. A. Frieman, and A. Gould, Signal modulation in cold dark matter detection, Phys. Rev. D 37, 3388 (1988).

[57] K. Freese, M. Lisanti, and C. Savage, Colloquium: Annual modulation of dark matter, Rev. Mod. Phys. 85, 1561 (2013).

[58] R. Bernabei et al. (DAMA Collaboration), The DAMA/ LIBRA apparatus, Nucl. Instrum. Methods Phys. Res., Sect. A 592, 297 (2008).

[59] F. James, MINUIT Function Minimization and Error Analysis: Reference Manual Version 94.1 (1994).

[60] F. Feroz, M. P. Hobson, and M. Bridges, MultiNest: An efficient and robust Bayesian inference tool for cosmology and particle physics, Mon. Not. R. Astron. Soc. 398, 1601 (2009).

[61] F. Feroz and M. P. Hobson, Multimodal nested sampling: An efficient and robust alternative to MCMC methods for astronomical data analysis, Mon. Not. R. Astron. Soc. 384, 449 (2008).

[62] F. Feroz, M. P. Hobson, E. Cameron, and A. N. Pettitt, Importance nested sampling and the MultiNest algorithm, Mon. Not. R. Astron. Soc. 384, 449 (2008).

[63] P. Scott, Pippi-painless parsing, post-processing and plotting of posterior and likelihood samples, Eur. Phys. J. Plus 127, 138 (2012). 